\title{
Análise teórica de desempenho térmico de motores de indução elétrica aletados
}

\author{
Theoretical analysis of thermal performance of electric motors \\ induction finned
}

\author{
${ }^{1}$ Ariane Silva Novais \\ ${ }^{2}$ Roberta Duarte Ferreira Chagas Comitra \\ 3 Élcio Nogueira
}

\footnotetext{
1 Graduada em Engenharia Mecânica pelo Centro Universitário de Volta Redonda - UniFOA; Mestranda em Meteorologia na Universidade Federal do Rio de Janeiro - UFRJ

2 Graduada em Engenharia Mecânica pelo Centro Universitário de Volta Redonda - UniFOA.

3 UERJ - Universidade do Estado do Rio de Janeiro.
}

\section{RESUMO}

Na indústria, os motores elétricos são largamente utilizados em função de sua simples construção e sua versatilidade, com relação à aplicação de cargas no mesmo. É muito comum o aquecimento excessivo do motor elétrico e sua consequente queima, devido à exposição a temperaturas ambientais elevadas e a ineficiência na dissipação do calor gerado por meio da diferença da potência útil fornecida pelo motor na ponta do eixo e a potência absorvida na linha de alimentação. 0 presente trabalho demonstra que esses modelos não remetem à realidade e aplica os dados de um motor elétrico, apresentando soluções analíticas para seu desempenho térmico, utilizando sistemas de aletas com perfis retangulares e condição de fluxo de calor por convecção prescrito na base. Efetua uma comparação entre o desempenho de dois materiais diferentes na composição da carcaça e das aletas do motor. São eles, alumínio e ferro fundido. Os resultados teóricos obtidos demonstram que não se pode negligenciar o efeito da resistência térmica interna na solução de desempenho térmico dos motores elétricos, e que essa resistência térmica deve ser objeto de estudo experimental detalhado, pois há escassez de resultados confiáveis na literatura.

\section{PALAVRAS-CHAVE}

Aletas; motores elétricos; desempenho térmico; alumínio e ferro fundido.

\begin{abstract}
In industry, the electric motors are widely used due to its simple construction and versatility with respect to the application of loads in it. It is very common overheating of the electric motor and its consequent burning, depending on exposure to elevated environmental temperatures and inefficiency in the dissipation of heat generated by the difference of the net power supplied by the motor shaft and the tip of the absorbed power on line supply. This work demonstrates that these models do not refer to reality and applies the data of an electric motor, presenting analytical solutions for thermal performance using fin systems with rectangular profiles and heat flux condition prescribed by convection at the base. Performs a comparison between the performance of two different materials carcass composition motor and fins, they are aluminum and cast iron. The theoretical results show that one can not neglect the effect of internal thermal resistance in thermal solution performance of electric motors, thermal resistance and that this should be the subject of detailed experimental study, since there is a shortage of reliable results in the literature.
\end{abstract}

\section{KEYWORDS}

Fins; electric motors; thermal performance; aluminium and cast iron.

\section{Como você deve citar?}

NOVAIS, A. S.; COMITRA, R. D. F. C.; NOGUEIRA, É. Análise teórica de desempenho térmico de motores de indução elétrica aletados. Cadernos UniFOA, Volta Redonda, n² 25, quadrimestral, p. 19-34, ago. 2014. 


\section{INTRODUÇÃO}

O motor elétrico é um equipamento que converte energia elétrica em energia mecânica de rotação, através de interações eletromagnéticas entre seus componentes, com a finalidade de promover movimento em outros corpos pelo giro de seu eixo. É o meio mais aplicado na conversão de energia para o acionamento das mais diversas cargas, no ramo industrial. Isso se dá, pelo fato de possuir componentes padronizados de fácil manutenção e reposição, simplicidade de comando, custo reduzido, elevado grau de automatização e grande flexibilidade no que diz respeito à adaptação aos mais variados tipos de cargas.

O princípio de funcionamento dos motores elétricos é baseado no campo magnético gerado ao redor de um condutor, quando este é percorrido por uma corrente elétrica. Existe uma ampla variedade de motores elétricos disponíveis no mercado e a adoção de um modelo deve combinar características mecânicas e elétricas adequadas ao processo, satisfazendo fonte de alimentação, condições ambientais, exigências da carga e condições de serviço.

Bonnett e Soukup (1992), num estudo sobre falhas em motores elétricos de indução, definem que as falhas ocorrem devido às seguintes razões: térmica, elétrica, mecânica e ambiental. De acordo com Cezário (2007), a elevação de temperatura é considerada um dos principais fatores prejudiciais ao desempenho e ao funcionamento de qualquer máquina elétrica girante, pelo fato de alterar as propriedades mecânicas e elétricas dos materiais, reduzir o intervalo de relubrificação dos mancais e, principalmente, afetar o desempenho dos materiais que compõem o sistema de isolamento elétrico. Dentre os componentes que constituem o motor elétrico de indução, os rolamentos e o sistema de isolamento elétrico são mais susceptíveis à influência da elevação de temperatura.

Segundo Almeida (2004), a camada isolante de um motor elétrico é um dos principais componentes no mesmo, pois ela isola eletricamente as várias partes da máquina e sua vida útil é considerada a vida útil do próprio motor. Muitos fatores interferem no envelhecimento do isolante, entretanto a maior causa é o sobreaquecimento. Por esse fato, se faz relevante que o motor elétrico opere com temperaturas compatíveis aos limites térmicos de seus isolamentos e que a taxa de dissipação de calor seja a maior possível nas condições de operação. De acordo com a Lei de Montsinger (1913), se a camada isolante for submetida a valores de temperatura de 8 a $10^{\circ} \mathrm{C}$ acima de sua temperatura limite, sua vida útil e, consequentemente, a vida útil do motor, terá uma redução da ordem de $50 \%$.

De acordo com Almeida (2004), o calor do motor é gerado pelas perdas do processo de conversão da energia elétrica em mecânica, decorrente da diferença entre a potência útil fornecida pela máquina elétrica na ponta do eixo e a potência absorvida da linha de alimentação. Ribeiro (2008), em sua dissertação de mestrado em engenharia mecânica, afırma que a máxima temperatura ambiente em que um motor padrão pode operar é de $40^{\circ} \mathrm{C}$.

Devido aos riscos oferecidos pela elevação de temperatura aos materiais que compõem o motor elétrico, torna-se de extrema importância a busca de mecanismos que facilitem ou até mesmo aumentem a dissipação de energia térmica do interior do motor para um ambiente externo. Lia e Quites (2006) afirmam que, para elevar a transferência de calor em um sistema, é preciso reduzir as resistências térmicas envolvidas no mesmo e isso pode ser concretizado com o aumento da velocidade de escoamento do fluido, reduzindo a espessura da parede, alterando o tipo de material da parede e alterando as dimensões da superfície com o uso de aletas. As aletas são superfícies estendidas, confeccionadas de material metálico que aumentam a área disponível para dissipação do calor no equipamento, auxiliando na troca de energia entre o meio e na consequente redução da temperatura do motor. 
A dissipação de energia térmica no motor elétrico é dependente da transferência de calor por convecção a qual o sistema está submetido, já que essa troca ocorre entre um fluído em movimento (ar) e a superfície do motor, que estão sob diferentes temperaturas. Esse fenômeno ocorre devido ao deslocamento molecular aleatório e ao movimento global do fluído regido pelo coeficiente de convecção.

O coeficiente de convecção é uma constante de proporcionalidade que leva em consideração a geometria da superfície, a natureza do escoamento, as propriedades e velocidade do fluído, a condutividade térmica do material envolvido, o acabamento superficial, a dureza, a temperatura média na interface e o fluxo de calor (Ordenes, 2008). Diante da dependência de tantas variáveis, Moreira (2012) afirma que a determinação do coeficiente de convecção se torna complexa e exige solucionar equações diferenciais da condução de calor.

Além da convecção atuante na região externa à carcaça do motor, e seu respectivo coeficiente de convecção, existe também na parte interna do motor, condução aliada à convecção. A interface de contato entre a parede do motor e seu isolamento é promovida por uma união mecânica que gera uma resistência térmica devido ao contato imperfeito entre os meios, fruto de pequenas rugosidades e ondulações. Nessa região ocorre condução nos pontos de contato e condução através dos fluidos aprisionados nos interstícios das rugosidades e ondulações. Dessa forma, é necessário determinar dois coeficientes de convecção, um deles referente à região externa e outro à interna da carcaça do motor, sendo esse último dependente ainda das imperfeições macroscópicas nas superfícies e da pressão de contato dos materiais, do fluido contido nos interstícios, do filme de óxidos aplicado e do calço metálico envolvido. Esse último coeficiente de convecção é, sem dúvida, o mais complexo dos dois processos e o mais difícil de se obter experimentalmente.

\section{OBJETIVO}

O objetivo do presente trabalho é fazer um estudo teórico do desempenho térmico de motores de indução elétrica providos de aletas retangulares, com fluxo de calor convectivo na base. Utilizamos como objeto de estudo um motor elétrico para o qual desenvolvemos soluções analíticas para perfis de temperatura, taxa de transferência de calor, eficiência e efetividade das aletas e do motor, considerando fluxo de calor convectivo constante prescrito na base do mesmo. 0 fluxo de calor prescrito foi simulado por meio de uma troca de calor por convecção entre o núcleo e a carcaça do motor. A simulação é factível, pois existe um espaço entre o núcleo e a carcaça ocupada pelo ar, produzindo uma resistência térmica na região. Dessa forma, pretende-se contribuir para os estudos da determinação de uma solução mais viável e compatível com a prática, que reduza a "queima" e consequente perda de motores elétricos no âmbito industrial.

\section{METODOLOGIA}

Desenvolvemos a solução analítica para a equação diferencial ordinária linear homogênea de segunda ordem destinada à determinação do perfil de temperatura e da taxa de transferência na carcaça de um motor elétrico. Com a modelagem matemática definida, aplicamos a linguagem ForTran - Fórmula Translation - para gerar inúmeros dados referentes ao perfil de temperatura e à taxa de transferência de calor desse sistema, quando submetido a diferentes coeficientes de transferência de calor por convecção, fazendo uso de distintos valores de temperatura do meio externo ao motor. De posse dos dados gerados, com o auxílio da ferramenta Grapher, desenvolvemos gráficos a fim de facilitar a análise dos dados obtidos. 


\section{DESENVOLVIMENTO}

De acordo com o catálogo do fabricante, estabelecemos, para efeito de análise, como temperatura máxima de trabalho na parede do motor, o valor de $98{ }^{\circ} \mathrm{C}$ e rendimento igual a $75 \%$. Dessa forma, podemos afirmar que $25 \%$ da potência gerada pelo motor é transformada em energia térmica, que se não dissipada adequadamente, aquecerá o motor, promovendo sua queima. 0 motor possui 32 aletas de ferro fundido para auxiliar no processo de dissipação de energia térmica. Sendo assim, cada aleta deve dissipar acima de 5,85 Watts, em regime permanente, para que o sistema funcione de maneira satisfatória. A seguir, na Tabela 1, temos alguns dados complementares do motor em questão.

Tabela 01 - Dados complementares do Motor.

\begin{tabular}{|l|l|}
\hline Dados do Motor & $1 \mathrm{cv}$ \\
\hline Potência & 4 \\
\hline Número de pólos & $139,60 \mathrm{~mm}$ \\
\hline Diâmetro Externo do motor & $130,13 \mathrm{~mm}$ \\
\hline Largura do motor & $5,84 \mathrm{~mm}$ \\
\hline Largura da base da aleta & $17,00 \mathrm{~mm}$ \\
\hline Altura da aleta & 32 \\
\hline Número de aletas & $40^{\circ} \mathrm{C}$ \\
\hline Temperatura máxima desejável no ambiente de trabalho & \\
\hline
\end{tabular}

A Figura 1, a seguir, esquematiza uma aleta retangular no motor em análise.

Figura 1 - Representação esquemática de uma aleta retangular no motor em análise.

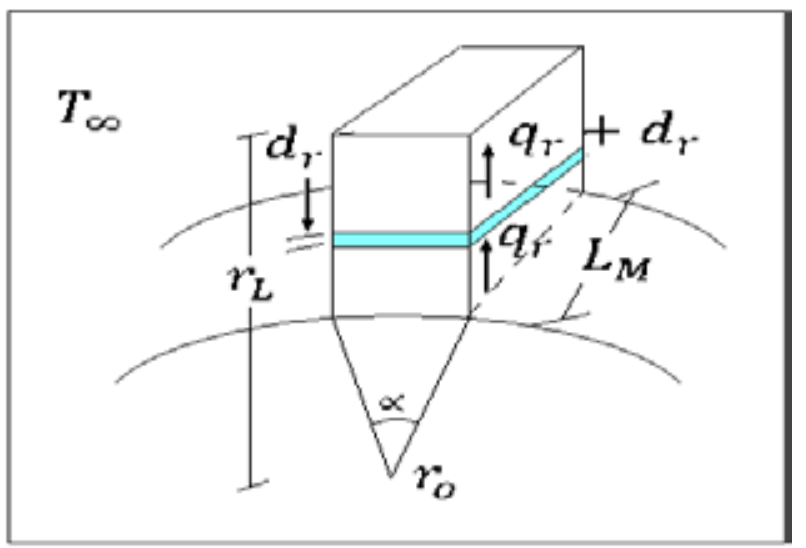

Temos a Equação Diferencial Ordinária (E.D.O.) descrita pela equação 01 abaixo e as condições de contorno aplicadas para o caso em análise, nas equações 02 e 03 , a seguir. 


$$
\frac{\partial^{2} T(r)}{d r^{2}}=\frac{h \cdot P}{k \cdot A_{b}} \cdot\left(T_{(r)}-T_{\infty}\right)
$$

$1^{\text {a }}$ Condição de Contorno: para $\mathrm{r}=0$

$-\frac{k \partial T}{d r}=h_{1}\left[T_{m}-T_{b}\right]$

$2^{\mathrm{a}}$ Condição de Contorno: para $\mathrm{r}=\mathrm{L}$

$-\frac{k \partial T}{d r}=h_{2}\left[T_{L}-T_{\infty}\right]$

Considerando $\theta_{\mathrm{r}}$ como temperatura auxiliar, conforme equação 04 , abaixo.

$\theta_{(r)}=T_{(r)}-T_{\infty}$

Temos $\theta_{M}$ e $\theta_{b}$ conforme as equações 05, 07 e 09.

$$
\theta_{(M)}=T_{(M)}-T_{\infty}
$$

$T_{(M)}=\theta_{(M)}-T_{\infty}$

$\theta_{(o)}=T_{(b)}-T_{\infty}$

$T_{(b)}=\theta_{(o)}-T_{\infty}$

$\theta_{(L)}=T_{(L)}-T_{\infty}$

$$
T_{(L)}=\theta_{(L)}+T_{\infty}
$$

Subtraindo a equação 05 da 07, temos:

$T_{(M)}-T_{b}=\theta_{(M)}-\theta_{o}$

Rearranjando as equações anteriores em função da temperatura auxiliar $(\theta)$, temos:

$\frac{d^{2} \theta_{(r)}}{d r^{2}}=\frac{h \cdot P}{k \cdot A_{b}} \cdot \theta_{(r)}$

$1^{\text {a }}$ Condição de Contorno: para $\mathrm{r}=0$ 
$-\frac{k \partial \theta}{d r}=h_{1}\left[\theta_{m}-\theta_{o}\right]$

$2^{\mathrm{a}}$ Condição de Contorno: para $\mathrm{r}=\mathrm{L}$

$-\frac{k \partial \theta}{d r}=h_{2} \cdot \theta_{L}$

Considerando:

$m^{2}=\frac{h \cdot P}{k \cdot A_{b}}$

Sendo, o perímetro da aleta, podemos reorganizar a equação 12 e obter a equação diferencial ordinária linear homogênea de segunda ordem, descrita abaixo.

$\frac{\partial^{2} \theta_{(r)}}{d r^{2}}=m^{2} \cdot \theta_{(r)}$

Como solução da EDO Linear Homogênea, temos o polinômio característico associado abaixo:

$\theta_{r}=C_{1} \cdot e^{m \cdot r}+C_{2} \cdot e^{-m \cdot r}$

Da $1^{\text {a }}$ Condição de Contorno, podemos concluir:

$-k\left(C_{1} \cdot m \cdot e^{m \cdot 0}-C_{2} \cdot m \cdot e^{-m \cdot 0}\right)=h_{1}\left[\theta_{M}-\left(C_{1}+C_{2}\right)\right]$

$-\frac{k \cdot m}{h_{1}} C_{1}+\frac{k \cdot m}{h_{1}} C_{2}=\theta_{M}-C_{1}+C_{2}$

$C_{1}\left(1-\frac{k \cdot m}{h_{1}}\right)=\theta_{M}-C_{2}\left(1+\frac{k \cdot m}{h_{1}}\right)$

$C_{1}=\frac{\theta_{M}-C_{2}\left(1+^{k \cdot m} / h_{1}\right)}{\left(1-^{k \cdot m} / h_{1}\right)}$

Da $2^{\mathrm{a}}$ Condição de Contorno, temos:

$-\frac{k}{h_{2}}\left(C_{1} \cdot m \cdot e^{m \cdot L}-C_{2} \cdot m \cdot e^{-m \cdot L}\right)=C_{1} \cdot e^{m \cdot L}+C_{2} \cdot e^{-m \cdot L}$

$-\frac{k \cdot m \cdot C_{1} \cdot e^{m \cdot L}}{h_{2}}+\frac{k \cdot m C_{2} \cdot e^{-m \cdot L}}{h_{2}}=C_{1} \cdot e^{m \cdot L}+C_{2} \cdot e^{-m \cdot L}$

$-C_{1} \cdot e^{m \cdot L}\left(1+\frac{k \cdot m}{h_{2}}\right)=C_{2} \cdot e^{-m \cdot L}\left(1-\frac{k \cdot m}{h_{2}}\right)$ 
$C_{2}=-\frac{C_{1} \cdot e^{2 m \cdot L}\left(1+\frac{k \cdot m}{h_{2}}\right)}{\left(1-\frac{k \cdot m}{h_{2}}\right)}$

Substituindo a equação 21 na 25, temos:

$C_{1}=\frac{\theta_{M}+\frac{C_{1} \cdot e^{2 m \cdot L}\left(1+{ }^{k \cdot m} / h_{2}\right)}{\left(1-k \cdot m / h_{2}\right)} \cdot\left(1+^{k \cdot m / h_{1}}\right)}{\left(1-k \cdot m / h_{1}\right)}$

$C_{1}\left(1-\frac{k \cdot m}{h_{1}}\right)=\theta_{M}+\frac{C_{1} \cdot e^{2 m \cdot L}\left(1+^{k \cdot m} / h_{2}\right)}{\left(1-k \cdot m / h_{2}\right)} \cdot\left(1+\frac{k \cdot m}{h_{1}}\right)$

$C_{1}\left(1-\frac{k \cdot m}{h_{1}}\right)\left(1-\frac{k \cdot m}{h_{2}}\right)=\theta_{M}\left(1-\frac{k \cdot m}{h_{2}}\right)+C_{1} \cdot e^{2 m \cdot L}\left(1+\frac{k \cdot m}{h_{2}}\right)\left(1+\frac{k \cdot m}{h_{1}}\right)$

$C_{1}\left[\left(1-\frac{k \cdot m}{h_{1}}\right)\left(1-\frac{k \cdot m}{h_{2}}\right)-e^{2 m \cdot L}\left(1+\frac{k \cdot m}{h_{2}}\right)\left(1+\frac{k \cdot m}{h_{1}}\right)\right]=\theta_{M}\left(1-\frac{k \cdot m}{h_{2}}\right)$

$C_{1}=\frac{\theta_{M}\left(1-\frac{k \cdot m}{h_{2}}\right)}{\left[\left(1-\frac{k \cdot m}{h_{1}}\right)\left(1-\frac{k \cdot m}{h_{2}}\right)\right]-\left[e^{2 m \cdot L}\left(1+\frac{k \cdot m}{h_{2}}\right)\left(1+\frac{k \cdot m}{h_{1}}\right)\right]}$

De acordo com o descrito acima, podemos definir a taxa de calor na base da aleta como, em $r=0$ :

$\dot{q}=-k \cdot A_{b} \frac{\partial T}{\partial r}$

$\dot{q}=-k \cdot A_{b}\left(C_{1} \cdot m \cdot e^{m \cdot 0}-C_{2} \cdot m \cdot e^{-m \cdot 0}\right)$

$\dot{q}=-k \cdot A_{b} \cdot m\left(C_{1}-C_{2}\right)$

Voltando a condição inicial da equação 04, temperatura auxiliar, podemos reformulá-la da seguinte forma:

$\theta_{(r)}=T_{(r)}-T_{\infty}$

$T_{(r)}=\theta_{(r)}+T_{\infty}$

$T_{(r)}=C_{1} \cdot e^{m \cdot r}+C_{2} \cdot e^{-m \cdot r}+T_{\infty}$

Portanto, temos desenvolvidas as equações que definem a taxa de transferência de calor e o comportamento da temperatura ao longo de uma aleta presente no motor, considerando fluxo de calor convectivo prescrito na base da mesma. 


\section{RESULTADOS E DISCUSSÃO}

Os resultados obtidos são referentes à solução analítica para o fluxo de calor convectivo prescrito na base de apenas uma das trinta e duas aletas existentes na carcaça do motor. Está descrito o comportamento da superfície estendida, quando a mesma é constituída de ferro fundido $\left(\mathrm{k}=80 \mathrm{~W} /\left(\mathrm{m}^{2} . \mathrm{K}\right)\right.$, assim como quando é composta de alumínio $\left(\mathrm{k}=237 \mathrm{~W} /\left(\mathrm{m}^{2} . \mathrm{K}\right)\right.$.

Nas Figuras 2, 3, 4 e 5, a seguir, temos a representação da taxa de transferência de calor ocorrida em aletas de alumínio e ferro fundido, quando submetidas a diferentes temperaturas do meio e coeficientes de transferência de calor por convecção $\left(\mathrm{h}_{2}\right)$ na região externa à carcaça do motor.

Figura 2 - Taxa de Transferência de Calor em aletas de alumínio e ferro fundido $\left[h_{2}=10 \mathrm{~W} / \mathrm{m}^{2} . K\right]$

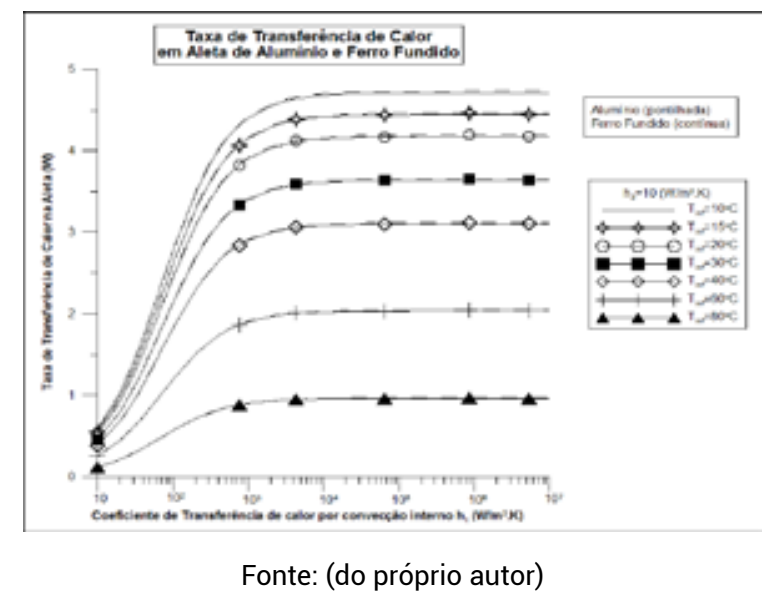

$\mathrm{Na}$ Figura 2, acima, a análise foi efetuada, considerando um coeficiente de transferência de calor na região externa ao motor $\left(\mathrm{h}_{2}\right)$ da ordem de $10(\mathrm{~W} / \mathrm{m} 2 . \mathrm{K})$. Dessa forma, foi possível visualizar que, para qualquer temperatura analisada, por maior que seja o $\mathrm{h}_{1}$, com um coeficiente de transferência de calor externo baixo, a taxa de transferência de calor será baixa e insuficiente para a retirada de calor necessária ao sistema. A Figura 2 também demonstra que, para valores de $\mathrm{h}_{1}$ acima de $10^{4}\left(\mathrm{~W} / \mathrm{m}^{2} . \mathrm{K}\right)$, o que temos reproduzida é a condição de temperatura prescrita na base da aleta. Também não foi perceptível a diferença entre a taxa promovida pela aleta de alumínio e a de ferro fundido. A Figura 3, apresentada a seguir, exibe a taxa de transferência de calor nas aletas quando submetidas a $u m h_{2}$ de $10^{2}\left(\mathrm{~W} / \mathrm{m}^{2} . K\right)$. 
Figura 3 - Taxa de Transferência de Calor em aletas de alumínio e ferro fundido $\left[h_{2}=10^{2} \mathrm{~W} / \mathrm{m}^{2} . \mathrm{K}\right]$

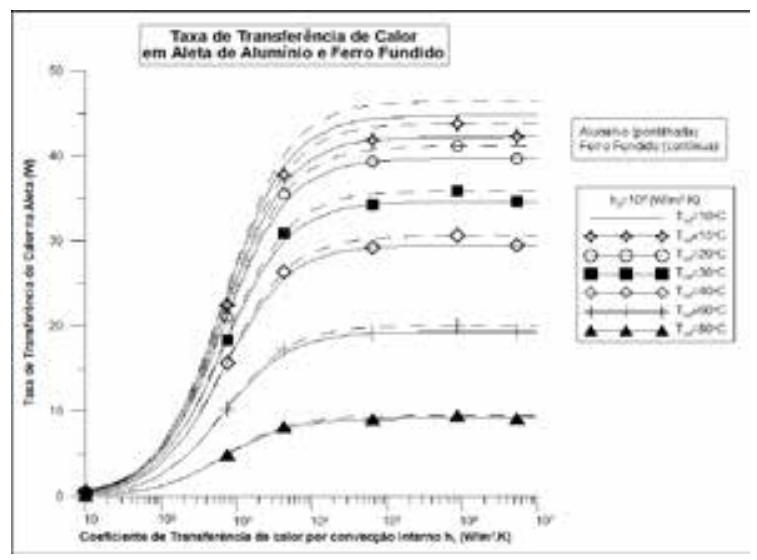

Fonte: (do próprio autor)

A Figura 3, representada acima, apresenta uma taxa de transferência de calor satisfatória, já na faixa compreendida após $\mathrm{h}_{1}=10^{2}\left(\mathrm{~W} / \mathrm{m}^{2} . \mathrm{K}\right)$. A temperatura ambiente mínima em que se é conveniente manter o motor sob condicionamento, devido aos custos de se promover o mesmo é de $15^{\circ} \mathrm{C}$, e a máxima suportada, como o próprio fabricante informa, é $40^{\circ} \mathrm{C}$. Podemos dizer que, para essa faixa limite de temperatura, com a aplicação de um coeficiente de transferência de calor na região externa ao motor $\left(\mathrm{h}_{2}\right)$ atuante de $10^{2}(\mathrm{~W} / \mathrm{m} 2 . \mathrm{K}), \mathrm{o} \mathrm{h}_{1}$ requerido para tornar o sistema satisfatório está entre $10^{2}$ e $10^{3}(\mathrm{~W} / \mathrm{m} 2 . \mathrm{K})$. Esses coeficientes de transferência de calor convectivos são de possível obtenção, quando associados aos sistemas de ventilação usualmente aplicados aos motores elétricos.

Nesse contexto, é possível perceber que a taxa de transferência de calor nas aletas de alumínio são levemente maiores que as apresentadas pelas aletas de ferro fundido, conforme esperado, em função de sua maior condutividade. Isso significa que a aleta de alumínio para trocar a mesma quantidade de energia na forma de calor que a aleta de ferro fundido troca, precisa de um coeficiente de transferência de calor numericamente menor.

A Figura 4, abaixo, exibe a taxa de transferência de calor nas aletas diante da aplicação de um $\mathrm{h}_{2}$ da ordem de $10^{3}\left(\mathrm{~W} / \mathrm{m}^{2} \cdot \mathrm{K}\right)$.

Figura 4 - Taxa de Transferência de Calor em aletas de alumínio e ferro fundido $\left[h_{2}=10^{3} \mathrm{~W} / \mathrm{m}^{2} \cdot K\right]$.

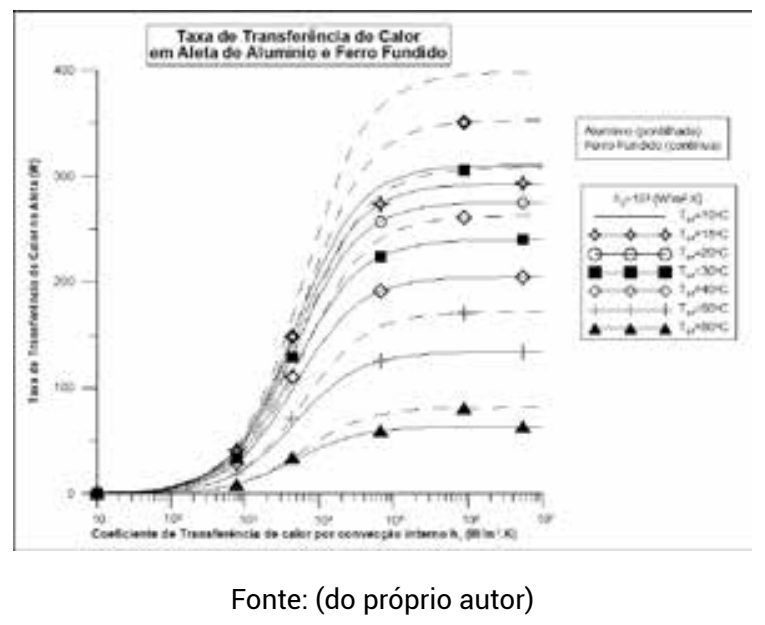


A Figura 4, acima, atingiu valores de taxa de transferência de calor de até 400 watts. Também é visível que, para a faixa de $\mathrm{h}_{1}$ compreendida após $10^{3}\left(\mathrm{~W} / \mathrm{m}^{2} . \mathrm{K}\right)$, existe um aumento significativo nos valores referentes às suas respectivas taxas de transferência de calor na aleta. Como os resultados apresentados são referentes a apenas uma das trinta e duas aletas existentes na carcaça do motor, fica evidente que a aplicação de um coeficiente de transferência de calor acima de $\mathrm{h}_{2}=10^{3}\left(\mathrm{~W} / \mathrm{m}^{2} \mathrm{~K}\right)$ se torna excessivo ao processo. Nessa Figura 4, é visível a diferença entre as taxas de transferência de calor produzidas pelos materiais em questão, assumindo o alumínio valores maiores.

A Figura 5, a seguir, exibe a taxa de transferência de calor no mesmo contexto apresentado anteriormente, porém com a aplicação de um coeficiente de transferência de calor na região externa ao motor $\left(\mathrm{h}_{2}\right)$ da ordem de $10^{4}\left(\mathrm{~W} / \mathrm{m}^{2} . \mathrm{K}\right)$.

Figura 5 - Taxa de Transferência de Calor em aletas de alumínio e ferro fundido $\left[h_{2}=10^{4} \mathrm{~W} / \mathrm{m}^{2} . K\right]$

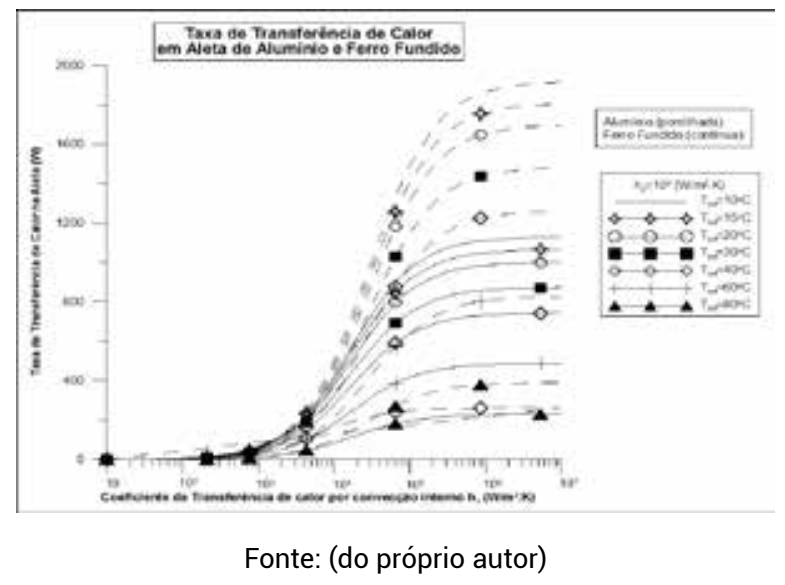

Os valores de taxa de transferência de calor apresentados na Figura 5, acima, em seu máximo, ultrapassa 1.600 watts. É também perceptível a maior taxa produzida pela aleta de alumínio. Isso se confirma com a análise da Figura 6, a seguir, que demonstra a relação entre a troca de calor efetuada por aletas de alumínio, quando comparadas às de ferro fundido, para diferentes coeficientes de transferência de calor externo $\left(h_{2}\right)$.

Figura 6 - Relação entre a Taxa de Transferência de Calor apresentada por aleta de alumínio quando comparada a da aleta de ferro fundido

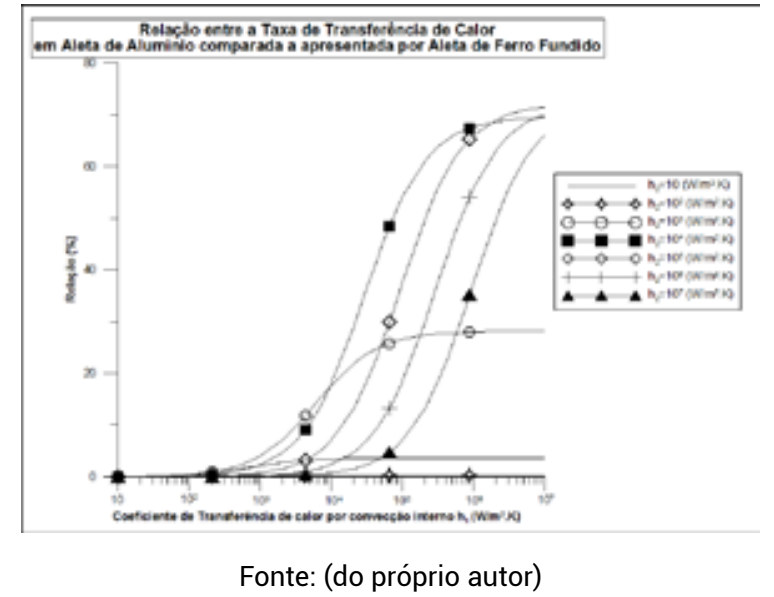


Analisando a Figura 6, acima, podemos ver a relação entre a taxa de transferência de calor apresentada pela aleta de alumínio e a apresentada pela aleta de ferro fundido. Nos casos analisados, a aleta de alumínio assumiu valores de taxa de transferência de calor até $71,45 \%$ maiores que os encontrados na de ferro fundido. Sendo tal relação potencializada quando o coeficiente de transferência de calor por convecção externo $\left(\mathrm{h}_{2}\right)$ assume valores entre $10^{3}$ e $10^{4}\left(\mathrm{~W} / \mathrm{m}^{2} . \mathrm{K}\right)$, indicando que o valor ótimo para a eficiência do alumínio sobre o ferro fundido está compreendido nessa faixa.

Além das análises efetuadas, também é possível concluir, por meio dos gráficos apresentados até o momento a dimensão da importância do coeficiente de transferência de calor externo $\left(h_{2}\right)$ adequado em um sistema onde se requer dissipação de calor. Com o objetivo de mensurar o desempenho da aleta, desenvolvemos a solução analítica para sua efetividade, sendo descrita conforme a Equação 37, abaixo.

$$
\text { Eff } f_{\text {aleta }}=\frac{\dot{q}_{\text {aleta }}}{h_{2} \times A_{b} \times \theta_{b}}
$$

Onde:

$$
\begin{aligned}
& \mathrm{q}_{\text {aleta }} \rightarrow \text { Taxa de calor trocada por cada aleta } \\
& \mathrm{A}_{\mathrm{b}} \rightarrow \text { Área da base da aleta } \\
& \theta_{\mathrm{b}} \rightarrow \text { Temperatura auxiliar na base da aleta } \\
& \mathrm{h}_{2} \rightarrow \text { coeficiente de convecção externo }
\end{aligned}
$$

Os resultados obtidos estão na Figura 7, a seguir. É importante informar que, durante o estudo da efetividade, a temperatura do meio ao qual o sistema foi submetida se mostrou independente, não influenciando na efetividade da aleta.

Figura 7 - Efetividade da aleta de alumínio e de ferro fundido

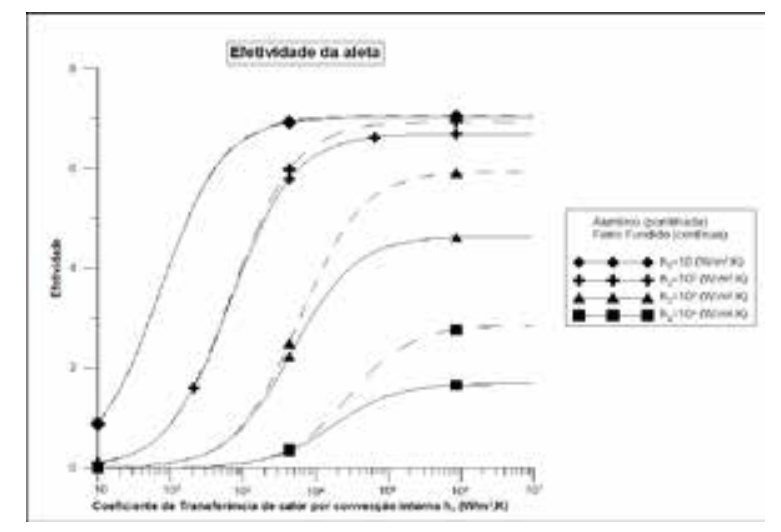

Fonte: (do próprio autor)

A Figura 7, apresentada acima, mostra que, a medida que se aumenta $h_{1}$, a efetividade também aumenta, para qualquer valor de $h_{2}$ aplicado. Para um dado $h_{1}$, quando $h_{2}$ é elevado, a efetividade tem seu valor reduzido, conforme representado pelo gráfico. Esse fato ocorre pois, quando o valor de $h_{2}$ é elevado, temos elevada também a troca de calor entre a aleta e o meio, o que faz com que a aleta tenda 
a ficar com a mesma temperatura do meio. Dessa forma, temos reduzido o gradiente de temperatura, o que reduz a efetividade.

Na Figura 7, que considera apenas a efetividade, é possível visualizar que o uso de aletas no motor em análise é justificado quando se opera com valores de $\mathrm{h}_{2}$ na faixa abaixo de $10^{3}\left(\mathrm{~W} / \mathrm{m}^{2} . \mathrm{K}\right)$, pois, nessas situações, a efetividade se mostrou igual ou maior que dois para os valores de $\mathrm{h}_{1}$ entre $10^{2}$ e $10^{3}\left(\mathrm{~W} / \mathrm{m}^{2} . \mathrm{K}\right)$. É observado, também, que a diferença entre os resultados apresentados pelos diferentes materiais não foi relevante. De acordo com o que foi exposto anteriormente, a aplicação de $u m \mathrm{~h}_{1}$ baixo, da ordem de $10\left(\mathrm{~W} / \mathrm{m}^{2} . \mathrm{K}\right)$, impõe uma elevada resistência entre o núcleo e a carcaça, impedindo a dissipação do calor gerado. Na Figura 7 , temos que, quando $h_{2}$ é submetido à $10\left(\mathrm{~W} / \mathrm{m}^{2} . \mathrm{K}\right)$, a aleta atinge uma efetividade equivalente a 2, com um $\mathrm{h}_{1}$ muito baixo, da ordem de $200\left(\mathrm{~W} / \mathrm{m}^{2} . \mathrm{K}\right)$. Sendo assim, podemos afirmar que apenas a efetividade da aleta ser maior que dois, não garante que o sistema terá uma dissipação adequada. Para que o motor tenha condições satisfatórias de trabalho é necessário adequar a taxa de transferência de calor obtida pela aleta, com sua efetividade e sua eficiência.

Visando fornecer mais um parâmetro de análise do sistema, desenvolvemos a solução teórica para a eficiência da aleta, que está descrita pela Equação 38, abaixo.

$$
\eta_{\text {aleta }}=\frac{\dot{q}_{\text {aleta }}}{h_{2} \times A_{T_{\text {aleta }}} \times \theta_{o}}
$$

Onde:

$\mathrm{A}_{\mathrm{T}}$ aleta $\rightarrow$ Área total de troca da aleta

De acordo com a Equação 38, a Figura 8, a seguir, foi gerada e exprime a eficiência de uma aleta. A eficiência, assim como a efetividade, independe da temperatura ambiente.

\section{Figura 8 - Eficiência da aleta de alumínio e de ferro fundido}

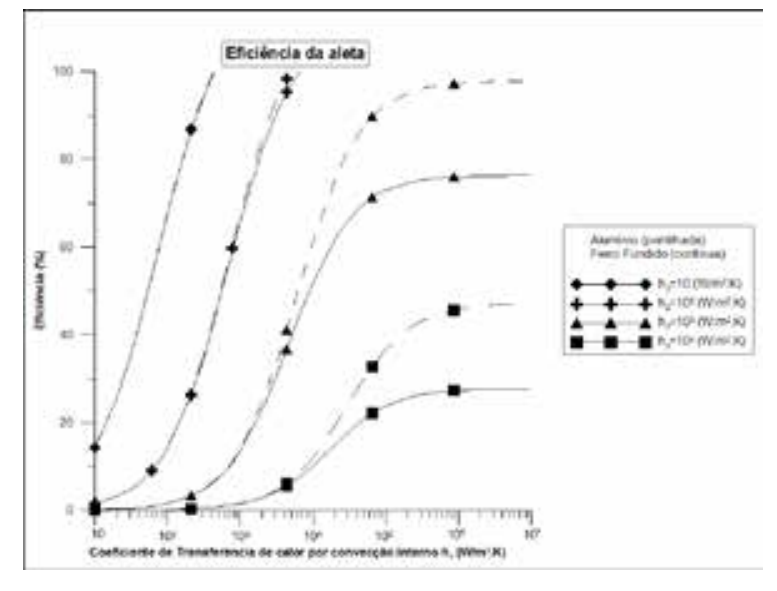

Fonte: (do próprio autor)

A Figura 8, acima, mostra que a medida que $\mathrm{h}_{1}$ tem seu valor elevado, a eficiência também é aumentada, em todas as situações. Fica claro, também, uma redução na eficiência do sistema, a medida que o valor de $\mathrm{h}_{2}$ aumenta. Isso ocorre devido à importância que o coeficiente de convecção externo 
tem no problema. Como vimos anteriormente, para o cálculo da eficiência, consideramos que a temperatura da base da aleta é a mesma ao longo de toda a sua superfície. Quanto maior o valor de $h_{2^{\prime}}$ maior é a propagação da temperatura do ambiente ao longo da aleta e, dessa forma, mais rapidamente é reduzido o gradiente de temperatura. Quanto menor o gradiente de temperatura, menor a eficiência da aleta no sistema. Também é possível dizer que a aplicação de alumínio ou ferro fundido proporcionou pequena ou nenhuma diferença nos resultados de eficiência apresentados, para a faixa de trabalho compreendida entre $10^{2}$ e $10^{3}\left(\mathrm{~W} / \mathrm{m}^{2} . \mathrm{K}\right)$, para ambos os coeficientes de transferência de calor, $\mathrm{h}_{1}$ e $\mathrm{h}_{2}$.

Conforme mostrado nos resultados apresentados, a faixa ideal de $h_{1}$ se encontra entre $10^{2}$ e $10^{3}$ $\left(\mathrm{W} / \mathrm{m}^{2} \cdot \mathrm{K}\right)$. Nessa faixa a eficiência da aleta se mostra mais vantajosa para baixos valores de $h_{2}$. Podemos

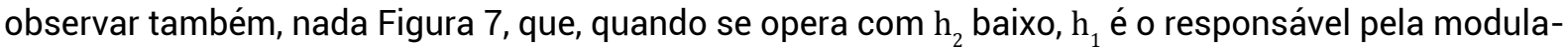
ção do problema. Para um mesmo $h_{2}$ quanto maior o $h_{1}$ aplicado, maior a eficiência. Porém, como, na prática, é improvável $\mathrm{h}_{1}$ com valores muito elevados, para se ter uma eficiência razoável, da ordem de $60 \%$, é viável e indicado a aplicação de um $\mathrm{h}_{1}$ na faixa entre $10^{2}$ e $10^{3}\left(\mathrm{~W} / \mathrm{m}^{2} . \mathrm{K}\right)$. Para confirmar o que foi apresentado acima e verificar a utilidade do uso de aletas nesse sistema, foi efetuada a análise da eficiência do motor, que pode ser traduzida pela relação entre a taxa trocada por todas as aletas e a taxa trocada por toda área do motor aletado na temperatura da base, de acordo com a Equação 39, abaixo.

$$
\eta_{\text {motor }}=\frac{32 \times \dot{q}_{\text {aleta }}}{h_{2} \times\left[\left(A_{T_{\text {motor }}}-32 \times A_{b}\right)+32 \times A_{T}\right] \times \theta_{b}}
$$

Onde:

$\mathrm{A}_{\mathrm{T}}$ motor $\rightarrow$ Área total de troca do motor

$$
\mathrm{A}_{\mathrm{T}} \rightarrow \text { Área total de troca da aleta }
$$

Os resultados de Eficiência apresentados pelo motor foram expressos na Figura 9, a seguir. Para fazer um comparativo entre os dados exibidos pelas aletas, estes também se encontram representados. Como não foi significativo o material aplicado, para o parâmetro em análise, optamos por manter apenas o material alumínio como constituinte.

Figura 9 - Eficiência do motor e da aleta - material de confecção: alumínio.

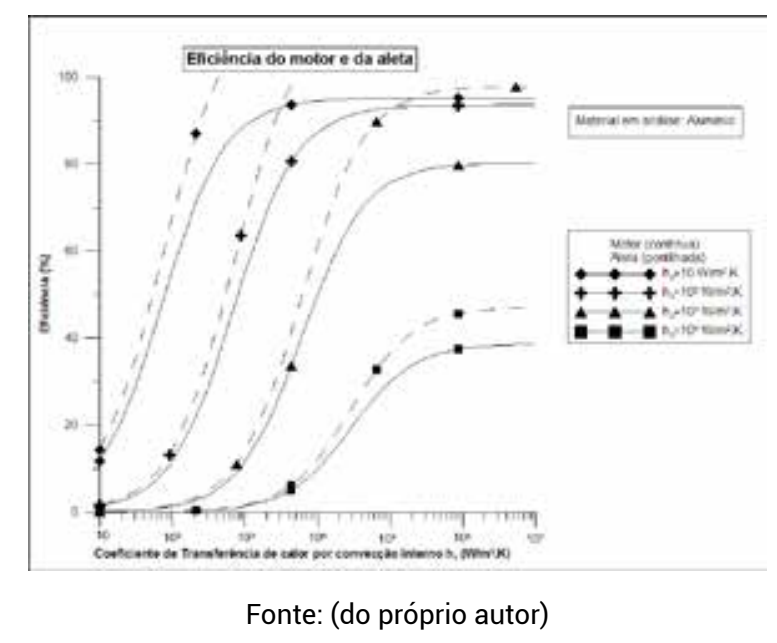

A Figura 9 nos permite visualizar que os resultados obtidos pela aleta são equivalentes aos apresentados pelo motor, em termos qualitativos. Entretanto, os valores absolutos para o motor são 
menores, conforme esperado, pois a área de troca disponível, quando se considera o motor como um todo, é superior às áreas das aletas somadas.

\section{CONCLUSÃO}

0 estudo apresentado mostrou que, cada uma das trinta e duas aletas, presentes no motor deve dissipar acima de 5,85 Watts, em regime permanente, para que o sistema funcione de maneira satisfatória.

Os resultados nos permitem confirmar a importância que o coeficiente de transferência de calor aplicado na região externa ao motor $\left(\mathrm{h}_{2}\right)$ tem na dissipação de calor sofrida pelo conjunto. Quando este assume um valor muito baixo, da ordem de $10\left(\mathrm{~W} / \mathrm{m}^{2} . \mathrm{K}\right)$, independente do valor estabelecido para 0 coeficiente de transferência de calor que atua na região interna do motor $\left(\mathrm{h}_{1}\right)$, a taxa de transferência de energia será baixa e insuficiente para a retirada do calor requerido. Quando temos elevada a condição de $h_{2}$ para $10^{3}\left(\mathrm{~W} / \mathrm{m}^{2} . \mathrm{K}\right)$, considerando a real faixa na qual a temperatura ambiente pode variar, entre 15 e $40^{\circ} \mathrm{C}, \mathrm{o} \mathrm{h}$ requerido para tornar a troca de calor no sistema satisfatória, está compreendido entre os valores de $10^{2}$ e $10^{3}\left(\mathrm{~W} / \mathrm{m}^{2} . \mathrm{K}\right)$. A utilização de valores acima de $10^{3}\left(\mathrm{~W} / \mathrm{m}^{2} . \mathrm{K}\right)$ para $\mathrm{h}_{2}$ se mostrou desnecessária, pois promoveu uma troca de calor excessiva. Além disso, aplicar um coeficiente tão elevado seria dispendioso e não condiz com a realidade.

Foi possível observar que $\mathrm{h}_{1}$ modula o problema através da resistência térmica que ele fornece ao conjunto. Essa resistência, localizada entre o núcleo e a carcaça do motor, é elevada quando o sistema está submetido a baixos valores de $\mathrm{h}_{1}$, da ordem de $10\left(\mathrm{~W} / \mathrm{m}^{2} . \mathrm{K}\right)$. Nessa condição, temos impedida a dissipação do calor gerado e, a aleta tende a permanecer por completo na temperatura do ambiente ao qual está exposta. Com a energia térmica "aprisionada", a temperatura interna do motor aumentará até sua queima e consequente inutilização. Quando o conjunto opera com valores de $h_{1}$ acima de $10^{4}\left(10^{7} \mathrm{~W} / \mathrm{m}^{2} . \mathrm{K}\right)$, a condição de temperatura prescrita na base da aleta é reproduzida, porém, como foi visto, o contato imperfeito entre a parede do motor e seu isolamento impede que essa situação se concretize nas condições reais de operação. 0 parâmetro efetividade nos mostrou que a inserção de aletas na carcaça do motor é justificada, quando $\mathrm{h}_{2}$ assume valores abaixo de $10^{3}\left(\mathrm{~W} / \mathrm{m}^{2} . \mathrm{K}\right)$. A eficiência de aleta apresentou valores aceitáveis, acima de $60 \%$, para a faixa de $h_{1}$ e $h_{2}$ compreendida entre $10^{2}$ e $10^{3}\left(\mathrm{~W} / \mathrm{m}^{2} . \mathrm{K}\right)$. Diante dos dados analíticos produzidos pelo presente trabalho, podemos dizer que a condição ideal de trabalho ocorre quando $h_{1}$ e $h_{2}$ assumem valores da ordem de $10^{2}$ a $10^{3}\left(\mathrm{~W} / \mathrm{m}^{2} . K\right)$.

Com relação aos materiais aplicados na confecção da carcaça e das aletas do motor, o alumínio só produzirá benefícios significativos quando o conjunto operar com ambos os coeficiente acima de $10^{3}\left(\mathrm{~W} / \mathrm{m}^{2} . \mathrm{K}\right)$. Esse resultado também pode ser comprovado no estudo "Solução analítica em aleta de perfil retangular: comparação de desempenho térmico entre alumínio e ferro fundido em motores elétricos", desenvolvido por Duarte, Novais e Nogueira (2012), no qual comprova-se que o alumínio atinge valores de taxa de transferência de calor muito maiores que os apresentados pelo ferro fundido, quando aplicado $h_{1}=10^{7} \mathrm{e}_{2}>10^{4}\left(\mathrm{~W} / \mathrm{m}^{2} . \mathrm{K}\right)$. Sugerimos a continuação deste trabalho, não só por meio do desenvolvimento de novos estudos teóricos, como também, de estudos experimentais para a determinação do coeficiente de convecção atuante na região compreendida entre o núcleo e a carcaça do motor, assim como da resistência térmica oferecida ao conjunto pela união mecânica da carcaça.

\section{Agradecimentos:}

A aluna Ariane Silva Novais agradece à Fundação Oswaldo Aranha - FOA pela bolsa de Iniciação Científica recebida durante a realização deste trabalho. 


\section{REFERÊNCIAS BIBLIOGRÁFICAS}

ALMEIDA, Antonio T. L. de. Motores Elétricos. Universicade Federal de Itajubá, agosto/2004.

ANEEL. Atlas de Energia Elétrica do Brasil. 1ed; 2002; p141.

BENG, Christopher Micallef; End Winding Cooling in Electric Machines. University of Nottingham , September 2006.

BONACORSO, NelsoGauze; NOLL, Valdir. Automação Eletropneumática. 11. ed. São Paulo: Érica, 2008.

CAMPANA, Saulo; Filho, Delly Oliveira; Soares, Antônio Alves; Oliveira, Rubens Alves de. Energia na Agricultura: Estudo comparativo de métodos para estimativa do carregamento e rendimento de motores elétricos para auditoria energética. Revista Brasileira de Engenharia agrícola e Ambiental, v.4, n.2, p.241 - 246, 2000. Campina Grande, PB, DEAg/UFPB.

CARDOSO, Rafael Balbino; NOGUEIRA, Luiz Augusto Horta; BARTONI, Edson da Costa; HADDAD, Jamil; SOUZA, Edson Palhares de; Avaliação da Economia de Energia Atribuídas a Ações de Etiquetagem Energética, em Motores de Indução no Brasil. Revista Brasileira de Energia, Vol.15, N. 1, 1 Sem. 2009, pp. 29-47.

CEZÁRIO, Cassiano A.; Análise do Escolamento de Ar em Motores de Indução Totalmento Fechados. Florianópolis, outubro de 2007.

DELLY Oliveira Filho I; Marcos C. Ribeiro II; Everardo C. Mantovani III; Antônio A. Soares IV; Haroldo C. FernandesVDelly, O. F.; Marcos, C. R.; Everardo C. M.; Antônio A. S.; Haroldo C. F.; Dimensionamento de Motores para o Bombeamento de Água. Eng. Agríc., Jaboticabal, v.30, n.6, p.1012-1022, nov./dez. 2010.

DIAS, Moisés de Mattos; SCHAEFFER, Lírio, DIAS, Arão de Matos; CÉSAR, José Lesina; Novas Perspectivas das Máquinas Elétricas Trifásicas a Partir da Utilização de Materiais Magnéticos Macios Sinterizados. Disponível em: http://www.liberato.com.br/upload/arquivos/0131010716421316.pdf. Acesso em: 03 de dezembro de 2013.

DUARTE, Denise Freire; NOVAIS, Ariane Silva; NOGUEIRA, Élcio. Solução Analítica em aleta de perfil retangular: comparação de desempenho térmico entre alumínio e ferro fundido em motores elétricos. UniFOA, Volta Redonda, 2012.

FILIPPO FILHO, G; Motor de Indução. São Paulo: Érica, 2000.

FORTRAN POWER STATION 4.0 - Microsoft Developer Studio - 1994-1995.

GRAPHER. Version 5.01, January 21, 2004.

INCROPERA, Frank P. et al.; Fundamentos de Transferência de Calor e de Massa. 6 ed. Rei de Janeiro: LTC, 2008. $644 \mathrm{p}$.

MERCURI, José Renato; MARTINS, Otávio Ferreira; TRAUTMANN, Paulo Victor; Desenvolvimento de um Sistema Supervisório para Análise de Motores Elétricos Através da Vibração Mecânica. Universidade Tecnológica Federal do Paraná, Curitiba, novembro de 2011. 
MINISTÉRIO DE MINAS E ENERGIA. Portaria Interministerial N 553. Disponível em: <http://www.inmetro. gov.br/qualidade/pdf/Portaria_MME_MCT_MDIC_553-2005[1].pdf>. Acesso em: 20 de maio de 2014.

MONTSINGER V. M., Loading Transformers by Temperature, AIEE Transaction, vol.32, 1913.

MOREIRA, José R.; Processos de Transferência de Calor. USP, set/2012.

OLIVEIRA, João Gabriel S. M.; Materiais Usados na Construção de Motores Elétricos. Porto Alegre, 2009.

ORDENES M.; Lambert R.; Güths, S.; Transferência de Calor Envolvente na Edificação. Departamento de Engenharia Civil - Universidade Federal de Santa Catarina, março/2008.

QUITES, Eduardo E. C. Lia, Luiz Renato B.; Introdução à Transferência de Calor. UFRSA, 2006.

REIS, Mara N. E.; Fenômeno de Transporte. PUC Minas, 2008.

SANTOS, Rafael Simões dos. Distúrbios nas Partidas de Motores de Indução Trifásicos. Viçosa, 2011.

SOUZA, Hamilton M. de; LEONELLI, Paulo A.; PIRES, Carlos A. P.; JÚNIOR, Valdir B. S.; PEREIRA, Roberto W. L.; Reflexões Sobre os Principais Programas em Eficiência Energética Existentes no Brasil. Revista Brasileira de energia, Vol. 15, N. 1, 1 Sem. 2009, pp. 7-26.

VALADEZ, José A. M.; Transferência de Calor. 2ª ed. Editora Alfaomega, 2002.

VOIGDLENER, Thiago; Escoamento e Transferência de Calor em Motores Elétricos de Indução. Universidade de Santa Catarina, Florianópolis, fevereiro de 2004.

WEG. 22 Maneiras de Melhorar a Eficiência do Motor e Prolongar sua Vida Útil. Disponível em: <http:// ecatalog.weg.net/files/wegnet/WEG-w22-motor-trifasico-22-ways-mercado-brasil-50024510-catalogoportugues-br.pdf>. Acesso em: 20 de maio de 2014. 